\title{
Dietary Aloe Vera Gel Powder and Extract Inhibit Azoxymethane- induced Colorectal Aberrant Crypt Foci in Mice Fed a High- fat Diet
}

\author{
Takeshi Chihara ${ }^{1 *}$, Kan Shimpo ${ }^{1}$, Takaaki Kaneko ${ }^{1}$, Hidehiko Beppu${ }^{1}$, Takashi \\ Higashiguchi $^{2}$, Shigeru Sonoda ${ }^{1}$, Miyuki Tanaka ${ }^{3}$, Muneo Yamada $^{3}$, Fumiaki Abe $^{3}$
}

\begin{abstract}
Aloe vera gel exhibits protective effects against insulin resistance as well as lipid-lowering and anti-diabetic effects. The anti-diabetic compounds in this gel were identified as Aloe-sterols. Aloe vera gel extract (AVGE) containing Aloe-sterols has recently been produced using a new procedure. We previously reported that AVGE reduced large-sized intestinal polyps in Apc-deficient Min mice fed a high fat diet (HFD), suggesting that Aloe vera gel may protect against colorectal cancer. In the present study, we examined the effects of Aloe vera gel powder (AVGP) and AVGE on azoxymethane-induced colorectal preneoplastic aberrant crypt foci (ACF) in mice fed a HFD. Male C57BL/6J mice were given a normal diet (ND), HFD, HFD containing $0.5 \%$ carboxymethyl cellulose solution, which was used as a solvent for AVGE (HFDC), HFD containing 3\% or 1\% AVGP, and HFDC containing $0.0125 \%$ (H-) or $0.00375 \%$ (L-) AVGE. The number of ACF was significantly lower in mice given $3 \%$ AVGP and H-AVGE than in those given HFD or HFDC alone. Moreover, 3\% AVGP, H-AVGE and L-AVGE significantly decreased the mean Ki-67 labeling index, assessed as a measure of cell proliferation in the colonic mucosa. In addition, hepatic phase II enzyme glutathione $S$-transferase mRNA levels were higher in the H-AVGE group than in the HFDC group. These results suggest that both AVGP and AVGE may have chemopreventive effects on colorectal carcinogenesis under the HFD condition. Furthermore, the concentration of Aloe-sterols was similar between 3\% AVGP and H-AVGE, suggesting that Aloe-sterols were the main active ingredients in this experiment.
\end{abstract}

Keywords: Aloe vera gel extract (AVGE) - Aloe-sterols - aberrant crypt foci (ACF) - high-fat diet (HFD)

Asian Pac J Cancer Prev, 16 (2), 683-687

\section{Introduction}

Aloe barbadensis Miller (Aloe vera) is one of approximately 420 Aloe species belonging to the family Liliaceae. Aloe vera is widely used for its therapeutic properties. It contains two major parts: leaves containing high concentrations of anthraquinone compounds, which have been used as a cathartic, and a clear and semisolid gel that has been used as a food and to treat burns and other wounds (Boudreau and Beland, 2006). Over 75 active ingredients have been identified in Aloe vera gel (Misawa et al., 2012a), and extracts from this gel were found to exhibit protective effects against insulin resistance in mice (Pérez et al., 2007) as well as lipidlowering effects in streptozotocin-induced diabetic rats (Rajasekaran et al., 2006). Anti-diabetic effects have also been reported in type 2 diabetic model mice, and the antidiabetic compounds were identified as five phytosterols, Aloe-sterols, namely lophenol, 24-methyl-lophenol, 24-ethyl-lophenol, cycloartanol, and 24-methylenecycloartanol (Tanaka et al., 2006; 2012). A previous study demonstrated that lophenol and cycloartanol acted as ligands of peroxisome proliferator-activated receptors (PPAR) $\alpha$ and $\gamma$, which regulate the metabolism of glucose and lipids in the livers of a diet-induced obesity mouse model (Nomaguchi et al., 2011). The administration of lophenol and cycloartanol have been shown to improve hyperglycemia and glucose metabolism and also reduced intra-abdominal fat accumulation in Zucker diabetic fatty (ZDF) rats fed a high-fat diet (Misawa et al., 2008). These findings have been attributed to the down-regulation of fatty acid synthesis and slight up-regulation of fatty acid oxidation in the liver with the administration of lophenol and cycloartanol (Misawa et al., 2008), thereby suggesting that Aloe vera gel could reduce the higher risk of colon cancer associated with fat intake as reported by epidemiological studies (Day et al., 2013).

We previously found that whole leaf Aloe arborescens

${ }^{1}$ Division of Biochemistry, Fujita Memorial Nanakuri Institute, Fujita Health University, Tsu, Mie, ${ }^{2}$ Department of Surgery and Palliative Medicine, School of Medicine, Fujita Health University, Dengakugakubo, Toyoake, Aichi, ${ }^{3}$ Functional Food Research Department, Food Science and Technology Institute, Morinaga Milk Industry, Higashihara, Zama, Kanagawa, Japan *For correspondence: tchihara@fujita-hu.ac.jp 
Miller var. natalnsis Berger (Kidachi aloe in Japanese), a different type of Aloe vera, inhibited the development of azoxymethane-induced aberrant crypt foci (ACF), which were identified as putative preneoplastic lesions in the rat colorectum (Shimpo et al., 2001). We also demonstrated that Kidachi Aloe inhibited the activation of phase I detoxification enzyme cytochrome P450 2E1 (CYP2E1) (Shimpo et al., 2003), activated the phase II detoxification enzyme quinone reductase (Shimpo et al., 2001), and reduced DNA adduct formation as a mechanism underlying the inhibition of ACF formation (Shimpo et al., 2003).

Aloe vera gel extract (AVGE) has recently been produced using a new procedure with supercritical carbon dioxide fluid, showing that AVGE contains Aloe-sterols (Tanaka et al., 2012). AVGE is nongenotoxic and does not cause any obvious side effects (Tanaka et al., 2012). We previously repored that AVGE reduced large-sized intestinal polyps in Apc-decifient Min mice fed a high-fat $\operatorname{diet}$ (Chihara et al., 2013), suggesting that AVGE protected against colonic inflammation and colorectal cancer. The treatment of Min mice with a high-fat diet was shown to increase the number of polyps (Day et al., 2013).

In the present study, we examined the effects of Aloe vera gel powder (AVGP) and AVGE on AOM-induced colorectal ACF in C57BL/6J mice fed a high-fat diet.

\section{Materials and Methods}

\section{Animals}

Five-week-old male C57BL/6J mice were purchased from Japan SLC Inc. (Hamamatsu, Japan). They were kept in groups of one or two in plastic cages on woodchip bedding in an animal facility controlled at a temperature of $23 \pm 5^{\circ} \mathrm{C}, 60 \pm 5 \%$ humidity, and with a 12-h light/ dark cycle. Mice were fed a normal diet. The care and use of the animals was according to the Regulations for the Management of Laboratory Animals at Fujita Health University, which is accredited by the Japanese Association of Laboratory Animal Facilities of Public and Private Universities (JALAP). The experimental protocols were approved by the Institutional Animal Care and Use Committee of Fujita Health University (Permit No. N0141).

\section{Experimental design and diets}

AVGP and AVGE dissolved in $0.5 \%$ carboxymethyl cellulose (CMC) were provided by Morinaga Milk Industry Co. Ltd. (Zama, Japan). The experimental design was described previously by Endo et al. (2009). After one week of acclimatization, mice were divided into normal diet (ND), high-fat diet (HFD), HFD containing $0.5 \%$ CMC solution without AVGE (HFDC), 3\% AVGP, 1\% AVGP, high dose AVGE (H-AVGE), and low dose AVGE (L-AVGE) groups. The ND group was given an AIN-93G diet, the HFD group was given a modified high-fat AIN93G diet (41\% of energy as fat, Oriental Yeast Co., Ltd., Tokyo, Japan), the $3 \%$ or $1 \%$ AVGP group was given HFD containing $3 \%$ or $1 \%$ AVGP, respectively, the H-AVGE group was given HFD containing $0.0125 \%$ AVGE, and the L-AVGE group was given HFD containing $0.00375 \%$
AVGE. After one week, all mice were intraperitoneally administered azoxymethane (AOM) at $10 \mathrm{mg} / \mathrm{kg}$ body weight once a week for two weeks. At 12 weeks of age, they were anesthetized with Nembutal, exsanguinated via the heart into heparin-coated syringes, and carefully autopsied. The colorectum and liver were immediately removed. The total number of ACF, the Ki-67 labeling index, and crypt length in the colorectum were measured according to the following procedures. The livers were stored at $-80^{\circ} \mathrm{C}$ until the mRNA expression levels of the phase I detoxification enzyme CYP2E1 and phase II detoxification enzyme glutathione $S$-transferase (GST) were determined.

\section{Analysis of aberrant crypt foci}

The colorectum was flushed gently with saline to remove any fecal contents, opened longitudinally from the cecum to the anus, placed between two pieces of filter paper, and fixed in $10 \%$ neutral buffered formalin. The numbers of ACF was counted, as described by Bird (1987). To facilitate counting, the colorectum was stained with $0.2 \%$ methylene blue solution and placed on a slide glass with the mucosal surface facing upward and the numbers of ACF was counted by microscopy at a magnification of $\times 40$.

\section{Immunostaining for $\mathrm{Ki}-67$}

After counting ACF, the colorectum was embedded in paraffin for immunohistochemistry. Immunohistochemistry using the rat monoclonal antibody TEC-3 against the Ki67 antigen (DakoCytomation, Glostrup, Denmark) was performed as a cell proliferation marker. This staining procedure was performed according to the protocols described by Katsuki et al. (2006) with minor changes. Each section was deparaffinized by xylene and rehydrated with graded alcohol. After washing, the sections were retrieved in an autoclave in citrate buffer $(\mathrm{pH}$ 6.0) for 20 min at $120^{\circ} \mathrm{C}$. After washing, endogeneous peroxidase activity was blocked by incubating slides in absolute methanol containing $3 \%$ hydrogen peroxidase $\left(\mathrm{H}_{2} \mathrm{O}_{2}\right)$ for $15 \mathrm{~min}$ at room temperature. After washing, they were then incubated with the antibody Ki-67 at its working dilution of 1:100 for $30 \mathrm{~min}$. After washing, Histofine simple stain mouse MAX PO anti-rat (Nichirei Biosciences Inc., Tokyo, Japan) was used as a secondary antibody. After washing, the Diaminobenzidine (DAB) Substrate Kit (Nichirei Biosciences Inc., Tokyo, Japan) was used to visualize staining and sections were counterstained using hematoxylin. No primary antibody was applied on the control negative staining.

To determine immunostaining, the number of epithelial cells of the rectum was counted in 5 to 10 crypts per mouse by light microscopy using $\times 400$ magnification. The results were expressed as the labeling index by the percentage of positively stained cells relative to the total number of cells counted (at least 500).

\section{Measurement of crypt length}

After immunostaining, the exact absolute length of each colonic crypt was measured in $\mu \mathrm{m}$. To measure the length using an ocular micrometer, well orientated crypts 
were selected and from the base of the crypt to the lumen was measured in 5 crypts per mouse.

\section{RNA preparation and quantification of CYP2E1 and GST $m R N A s$ in livers}

Total RNA was isolated from the mouse liver using the High Pure RNA Tissue Kit (Roche Applied Science, Mannheim, Germany) according to the manufacturer's protocol. RNA concentrations were determined by absorbance at $260 \mathrm{~nm}$, and $1 \mu \mathrm{g}$ of RNA was reversedtranscribed using the Transcriptor Universal cDNAMaster (Roche Applied Science, Mannheim, Germany). cDNA was amplified by real-time PCR with the Fast SYBR Green master mix (Applied Biosystems, Foster City, CA, USA). Amplification was performed using the 7500 Fast Real-Time PCR system (Applied Biosystems, Foster City, CA, USA) using specific primers targeting each of the identified mRNAs (available upon request). Product purity for the PCR reactions was confirmed by examining melting curves for the presence of a single peak. Real-time PCR results were normalized using cyclophilin A as an internal control.

\section{Data analysis}

Data are presented as the mean \pm S.E. Statistical comparisons were made with the Student's $t$-test or two-way analysis of variance where appropriate. These analyses were performed using Instat version 3.0 for Windows (GraphPad Software, Inc., San Diego, CA, USA). Differences were considered to be significant at $\mathrm{p}<0.05$.

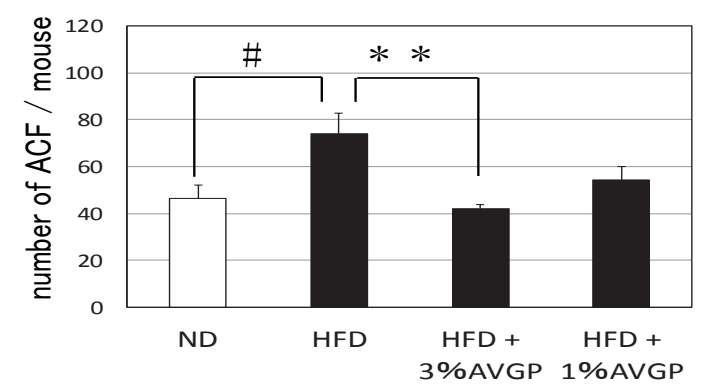

\section{Results}

\section{General observations}

Body weights during the experiment and the absolute and relative organ weights of the liver, kidney, and spleen remained unchanged in the ND, HFD, $1 \%$ AVGP, and $3 \%$ AVGP groups and ND, HFDC, L-AVGE, and H-AVGE groups, respectively. Food consumption was also unchanged (data not shown).

\section{Colorectal ACF}

$\mathrm{ACF}$ data are summarized in Figure 1. The number of ACF was significantly higher in mice given HFD alone than in those given ND. On the other hand, the number of ACF was significantly lower in the $3 \%$ AVGP group than in the HFD group (percentage inhibition: 43\%). The number of ACF was also slightly lower in 1\% AVGP group than in the HFD group (percentage inhibition: 27\%).

The number of ACF was significantly higher in mice given HFDC alone than in those given ND. On the other hand, the number of ACF was significantly lower in the H-AVGE group than in the HFDC group (percentage inhibition: $26 \%$ ). The number of ACF was slightly lower in the L-AVGE group than in the HFDC group (percentage inhibition: $9 \%$ ).

\section{Ki-67 labeling index}

The mean Ki-67 labeling indices are presented in Figure 2. The Ki-67 labeling index was significantly higher in the HFD group than in the ND group. On the other hand,

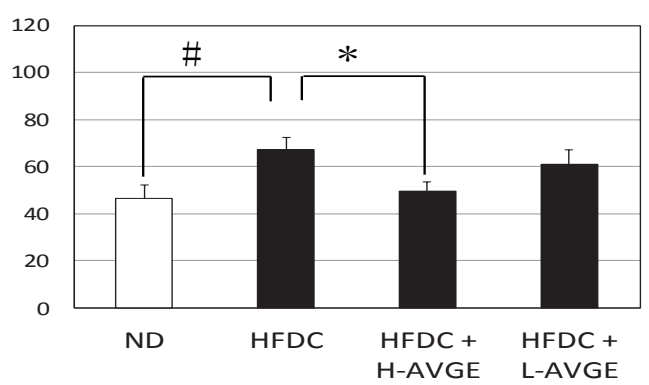

Figure 1. Effects of AVGP and AVGE on AOM-induced Colorectal ACF Formation in Mice Fed a High-Fat Diet. ACF formation was significantly greater in mice given a HFD than in those fed ND $\left({ }^{\#}<<0.05\right)$. In contrast, the number of ACF was significantly lower in mice given HFD $+3 \%$ AVGP and HFDC+H-AVGE than in those given HFD or HFDC alone $(* * \mathrm{p}<0.01$, ${ }^{*} \mathrm{p}<0.05$ ). ${ }^{*} \mathrm{p}<0.05$ ( $v s \mathrm{ND}$; unpaired t-test); ${ }^{*} \mathrm{p}<0.01$ ( $v s$ HFD; Dunn's multiple comparisons test); ${ }^{*} \mathrm{p}<0.05$ ( $v s$ HFDC; Dunnett's multiple comparisons test)
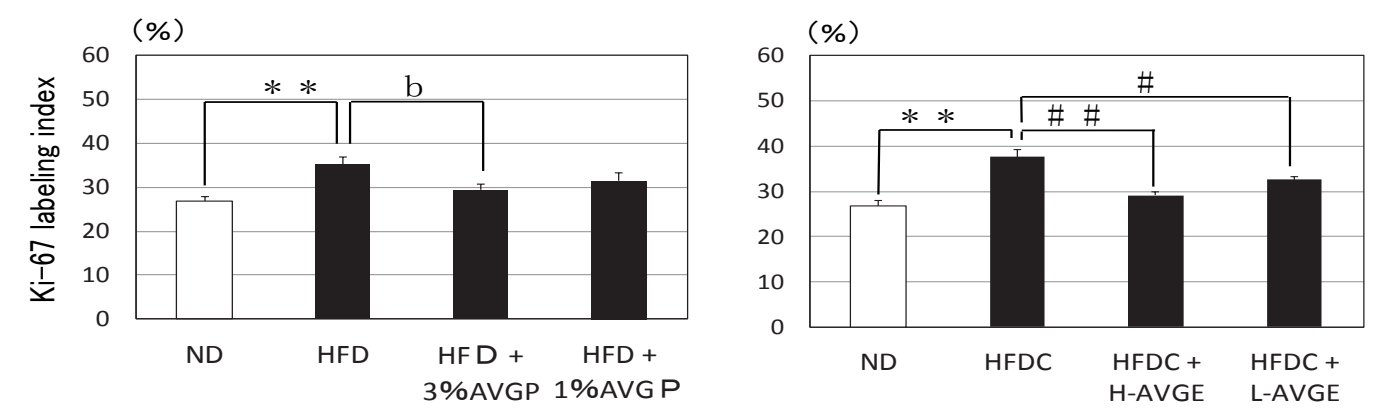

Figure 2. Ki-67 Labe ling Index in Normal Colorectal Mucosa. The mean Ki-67 labelling index was significantly higher in the HFD and HFDC groups than in the ND group $(* * \mathrm{p}<0.01)$. In contrast, it was significantly lower in the HFD+3\% AVGP, HFDC+H-AVGE, and HFDC+L-AVGE groups than in the HFD or HFDC groups $\left({ }^{b} \mathrm{p}<0.05,{ }^{\# \#} \mathrm{p}<0.01,{ }^{*} \mathrm{p}<0.05\right.$, Dunnett's multiple comparisons test 
it was significantly lower in the $3 \%$ AVGP group than in the HFD group (percentage inhibition: 17\%). The Ki-67 labeling index was slightly lower in the $1 \%$ AVGP group than in the HFD group (percentage inhibition: 11\%).

It was significantly higher in the HFDC group than in the ND group, but was significantly lower in the $\mathrm{H}$ - and L-AVGE groups than in the HFDC group (percentage inhibition: $22 \%$ and $13 \%$, respectively).

\section{Crypt length}

Crypt length was significantly longer in the HFD group than in the ND group (HFD group: $175.9 \pm 4.48 \mu \mathrm{m} v s$ ND group: $138.4 \pm 9.47 \mu \mathrm{m}, \mathrm{p}<0.05)$. However, it was slightly shorter in the 3\% AVGP and 1\% AVGP groups (3\% AVGP group: $161.6 \pm 5.05,1 \%$ AVGP group: $161.9 \pm 1.95)$. Crypt length was significantly longer in the HFDC group than in the ND group (HFDC group: $180.7 \pm 13.12 \mu \mathrm{m} v s \mathrm{ND}$ group were $138.4 \pm 9.47 \mu \mathrm{m}, \mathrm{p}<0.05)$. However, it was slightly shorter in the H-AVGE and L-AVGE groups (H-AVGE group: $160.6 \pm 6.08 \mu \mathrm{m}$, L-AVGE group: $174.0 \pm 13.1 \mu \mathrm{m})$.

\section{mRNA expression levels of CYP2E1 and GST}

No significant differences were observed in the mRNA expression levels of CYP2E1 between the HFDC and H-AVGE groups. However, the mRNA expression levels of GST were 1.61-fold higher in the H-AVGE group than in the HFDC group.

\section{Discussion}

Previous studies performed on animals described a relation between HFD consumption and the risk of colon cancer (Day et al., 2013). ACF are commonly accepted precursor lesions for colorectal cancer (Waly et al., 2014). In the present study, the number of ACF was higher in mice given HFD or HFDC than in those given ND, as described by Endo et al. (2009). In contrast, we showed that the number of ACF was significantly lower in mice given 3\% AVGP than in those given HFD alone. The number of ACF was also significantly lower in mice given $\mathrm{H}$-AVGE than in those given HFDC alone. However, the percentage inhibition of ACF differed between of $3 \%$ AVGP and H-AVGE groups (43\% and 26\%, respectively). The reasons for this were as follows. AVGP contains approximately $35 \%$ polysaccharides by the dry mass, and these have been shown to exhibit anti-inflammatory, antioxidative, and anti-tumor effects (Boudreau and Beland, 2006). These functions may also be associated with the decrease observed in the number of ACF. However, AVGE contain few polysaccharides (Tanaka et al., 2012).

We also demonstrated that the Ki-67 labeling index in the colorectum was reduced by AVGP and AVGE, whereas crypt length in the respective groups were not increased. Crypt length has been shown to reflect colon inflammation and mucosal cell proliferation (Kaya et al., 2007). Therefore, we considered the suppression of colonic epithelial cell proliferation by AVGP and AVGE to be part of the mechanism causing decreases in the number of ACF. The percentage inhibition of the Ki-67 labeling index in both groups was similar. The major component of
AVGE is known to be Aloe-sterols (Misawa et al., 2012a) .In this study, no significant differences were observed in the concentration of Aloe-sterols between 3\% AVGP and $\mathrm{H}-\mathrm{AVGE}$ or $1 \%$ AVGP and L-AVGE. Hence, we assumed that the reduction observed in the Ki-67 labeling index largely depended on the function of Aloe-sterols. We previously reported that $\mathrm{H}-\mathrm{AVGE}$ reduced large-sized intestinal polyps ( $>2.5 \mathrm{~mm}$ in diameter) in Min mice fed HFD (Chihara et al., 2013). The suppression of colonic epithelial cell proliferation by AVGE may have affect the results obtained.

Furthermore, we detected the mRNA expression of CYP2E1 and GST in the liver. GST mRNA expression levels were 1.61-fold higher in the H-AVGE group than in the HFDC group. AOM is a potent carcinogen that is used to induce colon cancer in rodents. AOM metabolized in the liver to methylazoxymethanol (MAM) by phase I detoxification enzymes such as CYP2E1. MAM then readily yields the ultimate carcinogenic metabolite, high reactive methyldiazonium ions for the methylation of DNA (Rosenberg et al., 2009). Thereafter, phase II detoxification enzymes conjugate and guide these ions from the body (Sunkara et al., 2008). Singh et al. (2000) reported that the fresh leaf pulp extract of Aloe vera significantly reduced the levels of phase I detoxification enzymes and increased those of phase II detoxification enzymes in the livers of mice. In the present study, the mRNA expression level of the phase II detoxification enzyme GST was found to be elevated. This function may also be associated with decreases in the number of ACF. On the other hand, no significant differences were observed in the mRNA expression levels of CYP2E1 between the H-AVGE and HFDC groups. Padidar et al. (2012) demonstrated that high-fat (60\% of energy as fat) feeding increased the development of AOM-induced ACF lesions and was associated with increased CYP2E1 mRNA expression levels in the livers of mice. However, we did not observe a relationship between the number of ACF and mRNA expression levels of CYP2E1, and this was partly attributed to differences in energy from fat in each experimental diet (60\% vs 40\%). In this study, we analyzed the mRNA expression levels of CYP2E1 and GST in the H-AVGE group only. We previously stated that the concentration of Aloe-sterols was equivalent between the AVGE and AVGP groups in this study. Therefore, the mRNA expression levels of the two enzymes in the $3 \%$ AVGP group were estimated to be similar to those in the H-AVGE group.

Misawa et al. (2012b) speculated that Aloe-sterols in bioactive molecules containing Aloe vera were the main active ingredients with anti-obesity effects in a high-fat diet-induced obesity rat model. Recent epidemiological studies have also reported that obesity may be a major risk factor in many cancers, especially cancers of the colon and breast (Sung et al., 2011). Lophenol and cycloartanol, two kinds of Aloe-sterols, reduced visceral fat accumulation and improved hyperlipidemia and hyperglycemia in ZDF rats fed a high-fat diet (Misawa et al., 2008). Furthermore, these of Aloe-sterols altered the expression levels of genes related to glucose and lipid metabolism, and ameliorated obesity-associated metabolic disorders 
in ZDF rats given a high-fat diet (Misawa et al., 2012b). In the present study, ACF percentage inhibition was smaller in the H-AVGE group than in the $3 \%$ AVGP group. However, the percentage inhibition of the Ki-67 labeling index was similar between the AVGP and AVGE groups. Furthermore, the mRNA expression level of the phase II detoxification enzyme GST in the liver was slightly higher in the H-AVGE group. Our results, in combination with the above findings, indicate that Aloe-sterols are the main active ingredients that inhibit the formation of ACF under the HFD condition.

In conclusion, 3\% AVGP and H-AVGE suppressed the formation of ACF in mice fed a high-fat diet. The inhibition of cell proliferation in the colon and increases in the mRNA expression levels of the phase II enzyme GST in the liver may be partly responsible for this effect. Moreover, Aloe-sterols are assumed to be the main suppressing ingredients that inhibit the formation of ACF. These results suggest that both AVGP and AVGE may exhibit chemopreventive activity on colorectal carcinogenesis under the HFD condition.

\section{Acknowledgements}

This study was supported by Research Grants from Morinaga Milk Industry Co., Ltd. to K.S.

\section{References}

Bird RP (1987). Observation and quantification of aberrant crypts in the murine colon treated with a colon carcinogen: preliminary findings. Cancer Lett, 37, 147-51.

Boudreau MD, Beland FA (2006). An evaluation of the biological and toxicological properties of Aloe barbadensis (Miller), Aloe vera. J Environ Sci Health C Environ Carcinog Ecotoxicol Rev, 24, 103-54.

Chihara T, Shimpo K, Beppu H, et al (2013). Reduction of intestinal polyp formation in Min mice fed a high-fat diet with Aloe vera gel extract. Asian Pac J Cancer Prev, 14, 4435-40.

Day SD, Enos RT, McClellan JL, et al (2013). Linking inflammation to tumorigenesis in a mouse model of highfat-diet-enhanced colon cancer. Cytokine, 64, 454-62.

Endo H, Hosono K, Fujisawa T, et al (2009). Involvement of JNK pathway in the promotion of the early stage of colorectal carcinogenesis under high-fat dietary conditions. Gut, $\mathbf{5 8}$, 1637-43.

Katsuki T, Hirata K, Ishikawa H, et al (2006). Aged garlic extract has chemopreventive effects on 1,2-dimethylhydrazineinduced colon tumors in rats. J Nutr, 136, 847S-51S.

Kaya E, Geylan A, Kara N, Güven H, Yildiz L (2007). The effect of L-glutamine on mucosal healing in experimental colitis is superior to short-chain fatty acids. Turk J Gastroenterol, 18, 89-94.

Misawa E, Tanaka M, Nomaguchi K, et al (2008). Administration of phytosterols isolated from Aloe vera gel reduce visceral fat mass and improve hyperglycemia in Zucker diabetic fatty (ZDF) rats. Obes Res Clin Pract, 2, 239-45.

Misawa E, Tanaka M,Nabeshima K, et al (2012a).Administration of dried Aloe vera gel powder reduced body fat mass in diet-induced obesity (DIO) rats. J Nutr Sci Vitaminol, 58, 195-201.

Misawa E, Tanaka M, Nomaguchi K, et al (2012b). Oral ingestion of Aloe vera phytosterols alters hepatic gene expression profiles and ameliorates obesity-associated metabolic disorders in Zucker diabetic fatty rats. J Agric Food Chem, 60, 2799-806.

Nomaguchi K, Tanaka M, Misawa E, et al (2011). Aloe vera phytosterols act as ligands for PPAR and improve the expression levels of PPAR target genes in the livers of mice with diet-induced obesity. Obes Res Clin Pract, 5, 190-201.

Padidar S, Farquharson AJ, Williams LM, et al (2012). High-fat diet alters gene expression in the liver and colon : Links to increased development of aberrant crypt foci. Dig Dis Sci, 57, 1866-74.

Pérez YY, Jiménez-Ferrer E, Zamilpa A, et al (2007). Effect of a polyphenol-rich extract from Aloe vera gel on experimentally induced insulin resistance in mice. Am J Chin Med, 35, 1037-46.

Rajasekaran S, Ravi K, Sivagnanam K, Subramanian S (2006). Beneficial effects of Aloe vera leaf gel extract on lipid profile status in rats with streptozotocin diabetes. Clin Exp Pharmacol Physiol, 33, 232-7.

Rosenberg DW, Giardina C, Tanaka T (2009). Mouse models for the study of colon carcinogenesis. Carcinogenesis, $\mathbf{3 0}$, 183-96.

Shimpo K, Chihara T, Beppu H, et al (2001). Inhibition of azoxymethane-induced aberrant crypt foci formation in rat colorectum by whole leaf Aloe arborescens Miller var. natalensis Berger. Phytother Res, 15, 705-11.

Shimpo K, Chihara T, Beppu H, et al (2003). Inhibition of azoxymethane-induced DNA adduct formation by Aloe arborescens var. natalensis. Asian Pac J Cancer Prev, 4 , 247-51.

Singh RP, Dhanalakshmi S, Rao AR (2000). Chemomodulatory action of Aloe vera on the profiles of enzymes associated with carcinogen metabolism and antioxidant status regulation in mice. Phytomedicine, 7, 209-19.

Sung M-K, Yeon J-Y, Park S-Y, Park JHY, Choi M-S (2011). Obesity-induced metabolic stresses in breast and colon cancer. Ann N Y Acad Sci, 1229, 61-8.

Sunkara R, Verghese M, Panala V, et al (2008). Chemopreventive potential of Cranberries on azoxymethane induced aberrant crypt foci in Fisher 344 male rats. Int J Cancer Res, 4, 52-60.

Tanaka M, Misawa E, Ito Y, et al (2006). Identification of five phytosterols from Aloe vera gel as anti-diabetic compounds. Biol Pharm Bull, 29, 1418-22.

Tanaka M, Yamada M, Toida T, Iwatsuki K (2012). Safety evalution of supercritical carbon dioxide extract of Aloe vera gel. J Food Sci, 71, 2-9.

Waly MI, Al-Rawahi AS, Riyami MA, et al (2014). Amelioration of azoxymethane induced-carcinogenesis by reducing oxidative stress in rat colon by natural extracts. $B M C$ Complement Altern Med, 14, 60. 